\title{
Rod Ellis, Gary Barkhuizen, Analysing Learner
}

Language

Oxford : Oxford University Press, 2005

Marie-Françoise Narcy-Combes

\section{CpenEdition}

\section{Journals}

Édition électronique

URL : http://journals.openedition.org/asp/631

DOI : 10.4000/asp.631

ISBN : 978-2-8218-0400-5

ISSN : 2108-6354

\section{Éditeur}

Groupe d'étude et de recherche en anglais de spécialité

\section{Édition imprimée}

Date de publication : 1 janvier 2005

Pagination : 165-166

ISSN : 1246-8185

\section{Référence électronique}

Marie-Francoise Narcy-Combes, "Rod Ellis, Gary Barkhuizen, Analysing Learner Language », ASp [En ligne], 47-48 | 2005, mis en ligne le 02 février 2010, consulté le 21 septembre 2020. URL : http:// journals.openedition.org/asp/631 ; DOI : https://doi.org/10.4000/asp.631

Ce document a été généré automatiquement le 21 septembre 2020.

Tous droits réservés 


\section{Rod Ellis, Gary Barkhuizen, Analysing Learner Language}

Oxford : Oxford University Press, 2005

Marie-Françoise Narcy-Combes

\section{RÉFÉRENCE}

Ellis, Rod and Gary Barkhuizen. 2005. Analysing Learner Language. Oxford : Oxford University Press. 404 p. ISBN 978-0194316347. 
Ce livre vient à point nommé pour compléter les outils à la disposition des jeunes chercheurs en linguistique appliquée et didactique des langues, comme des praticiens de terrain désireux de conduire une recherche-action. Comme souvent en ce qui concerne les ouvrages de Rod Ellis, il s'agit d'une somme: une étude diachronique des outils utilisés depuis les années soixante par les chercheurs en acquisition des langues pour l'analyse des productions écrites et orales des apprenants de langue. Ces outils ont évolué en fonction des questions de recherche apparues au fil du temps, et sont révélateurs de la complexité et de la variété du domaine. Ce travail fait apparaître la diversité des conceptions sur la nature et les implications de l'acquisition d'une L2 et incite à se garder de tout dogmatisme à cet égard.

Après un premier chapitre concernant le recueil des données et les moyens employés pour les obtenir, les auteurs explorent sur dix chapitres les différentes méthodes d'analyse des productions langagières des apprenants tant au niveau du contenu que de la forme. Reliant constamment théories et pratique, ils donnent pour chaque approche les fondements historiques et théoriques qui les sous-tendent, décrivent la méthode elle-même et illustrent leur propos de nombreux exemples pratiques. Pour terminer, et c'est la cerise sur le gâteau, ils proposent une tâche à réaliser, afin que le lecteur/ apprenant puisse s'approprier le savoir par l'action, en cohérence avec les théories de l'apprentissage par les tâches dont Rod Ellis s'est fait l'écho. Le dernier chapitre, rédigé par Michael Barlow (dont la contribution a été sollicitée par les auteurs comme spécialiste du domaine), concerne l'utilisation du traitement informatique des données pour l'analyse des productions langagières. L'ouvrage propose régulièrement des tableaux synthétiques clairs utiles au chercheur, tel que par exemple celui présenté à la page 11 , où les trois paradigmes normatif, interprétatif et critique sont examinés selon six composants: l'orientation théorique, l'objectif de la recherche, le champ de la recherche, les participants, le recueil et l'analyse des données.

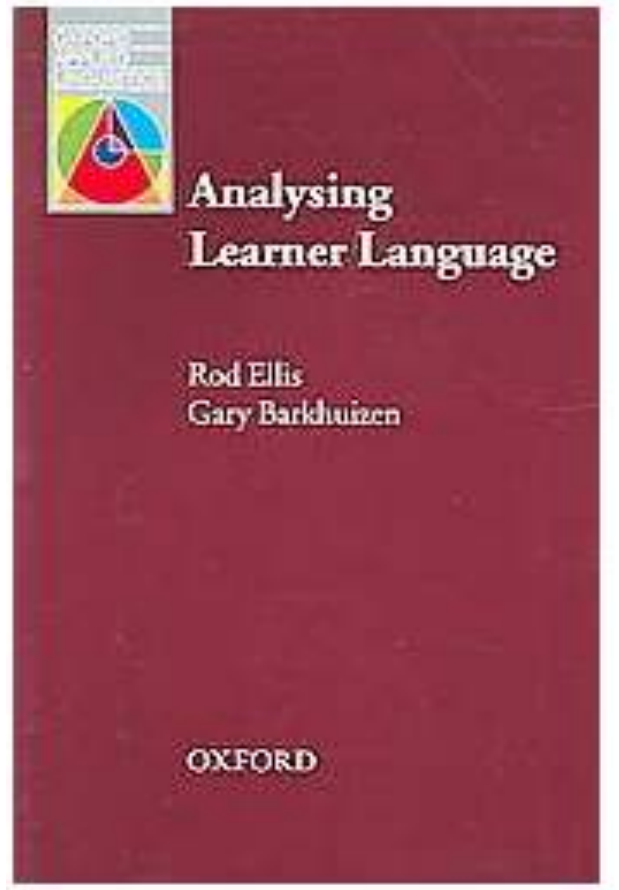

Les applications pratiques qu'offre le livre ne sont pas les moindres de ses atouts. Prenons quelques exemples pour le montrer. À la fin de la section 6 concernant l'analyse fonctionnelle, dont il faut rappeler que l'historique et les fondements théoriques ont été précisés tout au long du chapitre, ce qui en détermine également les limites, les auteurs proposent deux interactions au cours d'un jeu de rôle, la première entre un locuteur natif (américain) et un non natif (taïwanais), la seconde entre deux locuteurs natifs sur le même sujet: refuser un rendez-vous. La situation de communication est la suivante: un garçon invite la meilleure amie de son ex-petite amie à sortir avec lui ; elle refuse. La tâche consiste à identifier les stratégies utilisées 
par les locuteurs non natifs et les locuteurs natifs et de les comparer. Les auteurs suggèrent des étapes pour l'analyse: repérer les éléments du discours, décrire ces éléments, établir un tableau comparatif, commenter les résultats en fonction de la question de recherche. Ils invitent ensuite le lecteur à recueillir ses propres données pour l'analyse, sur le modèle proposé. En formation des maîtres ou en formation à la recherche, on mesure tout l'intérêt de cette tâche et de la discussion qui ne manquera pas de suivre, après que les participants se sont confrontés à la réalité des problèmes par l'action. Un peu plus loin, le chapitre 10 est consacré à l'analyse socioculturelle des interactions. La tâche s'appuie sur la transcription des interactions professeur/élève avec un apprenant adulte à Auckland en Nouvelle-Zélande et avec un élève de niveau intermédiaire dont la langue maternelle est un dialecte africain. Il s'agit d'étayage, c'est-à-dire du soutien que l'enseignant apporte à l'apprenant dans la construction de son interlangue. Les questions qui suivent invitent le lecteur à utiliser les outils méthodologiques décrits dans le chapitre pour analyser les deux interactions. Cette analyse les conduit à déterminer des éléments tels que le point linguistique qui fait l'objet de l'étayage, le type de relation pédagogique établie, les rapports de dominance, le type et le degré d'explicitation de l'aide prodiguée, le niveau de réussite des apprenants, et comment il peut s'expliquer en termes d'analyse socioculturelle. Ces deux exemples devraient donner une idée de l'étendue du champ et de la variété des approches proposées.

4 À travers cette rétrospective des différentes méthodes d'analyse des productions d'apprenants, ce livre offre une vue d'ensemble de l'état actuel du savoir en ce qui concerne l'apprentissage de L2. Il est une très bonne introduction à la méthodologie de la recherche en acquisition des langues même s'il ne se réclame pas de ce domaine, car il n'est pas possible d'analyser les productions des apprenants sans passer par les références théoriques qui permettent cette analyse.

\section{AUTEURS}

\section{MARIE-FRANÇOISE NARCY-COMBES}

Université de Nantes 